\title{
Predicting Traffic Accidents with Event Recorder Data
}

\author{
Yoshiaki Takimoto \\ NTT Service Evolution Laboratories \\ yoshiaki.takimoto.ar@hco.ntt.co.jp \\ Shuhei Yamamoto \\ NTT Service Evolution Laboratories \\ shuhei.yamamoto.ea@hco.ntt.co.jp
}

\author{
Yusuke Tanaka \\ NTT Service Evolution Laboratories \\ yusuke.tanaka.rh@hco.ntt.co.jp \\ Maya Okawa \\ NTT Service Evolution Laboratories \\ maya.ookawa.af@hco.ntt.co.jp
}

\author{
Takeshi Kurashima \\ NTT Service Evolution Laboratories \\ takeshi.kurashima.uf@hco.ntt.co.jp \\ Hiroyuki Toda \\ NTT Service Evolution Laboratories \\ hiroyuki.toda.xb@hco.ntt.co.jp
}

\begin{abstract}
Large amounts of data on accidents are continually being collected by dashboard cameras (dashcams). In this paper, we address the problem of predicting the occurrence of accidents: Our goal is to predict when accidents will occur based on stored dashcam data and analysis of live video streams. We propose a survival analysis model for predicting the event occurrence time. The occurrence of accidents involves changes in the situation of own car and surroundings. Therefore, the hazard function of the proposed model is modeled by a convolutional recurrent neural network that can capture it from high-dimensional time-series information, i.e., video. Another characteristic of our model is its incorporation of location data because how likely the events are to occur strongly depends on location. Our model can predict accidents by simultaneously considering video and location data. Experiments on real-world event recorder data show that our model can more accurately predict accident occurrences than baseline models.
\end{abstract}

\section{CCS CONCEPTS}

- Mathematics of computing $\rightarrow$ Survival analysis; $\bullet$ Computing methodologies $\rightarrow$ Neural networks; Computer vision.

\section{KEYWORDS}

event recorder data, traffic accident, survival analysis, recurrent neural network

\section{ACM Reference Format:}

Yoshiaki Takimoto, Yusuke Tanaka, Takeshi Kurashima, Shuhei Yamamoto, Maya Okawa, and Hiroyuki Toda. 2019. Predicting Traffic Accidents with Event Recorder Data. In 3rd ACM SIGSPATIAL International Workshop on Prediction of Human Mobility (PredictGIS'19), November 5, 2019, Chicago, IL, USA. ACM, New York, NY, USA, 4 pages. https://doi.org/10.1145/3356995. 3364535

\section{INTRODUCTION}

Due to the proliferation of dashboard cameras (dashcams), society is now generating large-scale volumes of driving data. The driving

Permission to make digital or hard copies of all or part of this work for personal or classroom use is granted without fee provided that copies are not made or distributed for profit or commercial advantage and that copies bear this notice and the full citation on the first page. Copyrights for components of this work owned by others than ACM must be honored. Abstracting with credit is permitted. To copy otherwise, or republish, to post on servers or to redistribute to lists, requires prior specific permission and/or a fee. Request permissions from permissions@acm.org.

PredictGIS'19, November 5, 2019, Chicago, IL, USA

(C) 2019 Association for Computing Machinery.

ACM ISBN 978-1-4503-6964-0/19/11 . .\$15.00

https://doi.org/10.1145/3356995.3364535

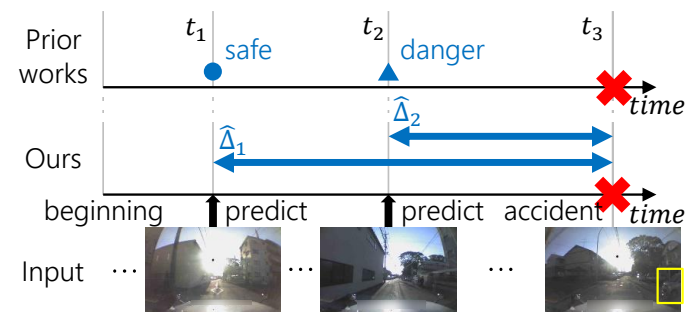

Figure 1: Task of prior works and ours. Output example of prior works and ours at $t_{1}$ and $t_{2}$. Both receive the same video stream from the beginning to $t_{1}$ or $t_{2}$ as input (an accident between own car and the car shown in the yellow box will occur at $t_{3}$ ). Prior works output safe at $t_{1}$ and danger at $t_{2}$. Ours outputs $\hat{\Delta}_{1}=t_{3}-t_{1}$ at $t_{1}$ and $\hat{\Delta}_{2}=t_{3}-t_{2}$ at $t_{2}$ indicating the lead time of the next event.

data involved in the event such as accident is called event recorder data. Event recorder data consists of a front video and location and provides a highly useful record of the process leading to the events (i.e., accidents). The accidents present in event recorder data exhibit a wide variety of circumstances, e.g., urban areas and rural roads, and causes for pedestrians and cars. Therefore, by utilizing the large amounts of event recorder data available, we hope to predict and thus stop accidents likely the occur in various situations. The realization of accident prediction will yield advanced driver assistance systems (ADAS), which prevent traffic accidents by automatic responses (e.g., sudden brake and rapid turn).

In this paper, we address the task of predicting the lead time of an accident between the car and another traffic obstacle based on the video stream of the dashcam. Solving this task allows ADAS to take appropriate action according to the lead time to the accident. For example, if the lead-time is 1 second later, sudden braking is required, but if it is 6 seconds later, alerting is sufficient. Several works $[1,11]$ tackled to anticipate the occurrence of accidents by using dashcam data. However, they do not estimate the lead time as shown in the upper part of Figure 1. In other words, they output only safe or danger and do not distinguish the lead time of 1 second and that of 6 seconds. That makes it extremely difficult to choose the most appropriate response.

We propose a survival analysis based model for predicting event occurrence time. Survival analysis provides a powerful way to model event occurrence; it enables us to estimate the lead time of the next event as shown in the lower part of Figure 1, by using both historical data including accidents, and right-censored data (i.e., no accident events are observed before the end of the observation period). To identify the video cues that point to a future accident, we use the convolutional recurrent neural network (CRNN) for 
capturing time-series changes of own car and the surrounding environment. The CRNN consists of the convolutional neural network $(\mathrm{CNN})$ and the recurrent neural network (RNN). In addition to the time-series changes, found in the dashcam video stream, our model takes account of the fact that accident occurrence also depends on location characteristics (e.g., the population of children and difference in driving style). Such information is extremely helpful in accurately estimating the lead time to the next event.

In summary, our contributions are as follows: (1) To the best of our knowledge, this is the first attempt to predict a traffic accident from event recorder data with survival analysis; (2) we propose a survival analysis model that inputs video stream and locations, the parameters of which can be learned using standard backpropagation algorithms with the negative log-likelihood loss function; (3) we show that our model can accurately predict the event occurrence time by effectively utilizing video and location data through experiments on real-world data.

\section{RELATED WORKS}

Anticipating Accidents from Event Recorder Data. A related task of anticipating accidents from event recorder data has been addressed recently in few studies. Here we introduce two representative studies $[1,11]$. To accurately anticipate collision between traffic objects in front video, Chan et al. proposed the dynamic spatial attention model, which has a deep neural network (DNN) architecture for appropriately handling object features such as object detection results obtained from each image [1]. Suzuki et al. tackled the task of anticipating accidents between own car and other objects, and they proposed a new loss function based on curriculum learning [11]. Although their models can determine whether an accident will occur within a few seconds, the exact time of accident occurrence is not output, that is, their goal is the binary classification of whether or not an accident will occur.

In this paper, we try to predict the time of the next accident with outputs such as "accident occurs after 3 seconds". This task is more challenging and more important than existing tasks because it is necessary to output a specific value to the most effective response.

Survival Analysis. Survival analysis can analyze the lead time of a specific event (such as death or malfunction that occurs at most once). It has the advantage of being able to consider the case where no event occurs during the observation period. There have been many survival models combined with DNN, which are advantageous in that they can increase the expressiveness of the hazard function $[6,12,14,15]$. Zhu et al. proposed a survival model called DeepConvServ [14]. By modeling the hazard function as CNN outputs, it can estimate the patient's lifespan from medical images. However, these existing models do not handle time-series information $[12,14,15]$; thus the performance is limited in such a situation that the time-series changes in the video stream are strongly relevant for predicting events. Jing et al. incorporated RNN into survival analysis for handling time-series data [6]. This model can consider a sequence of events, however, they cannot handle high-dimensional time-series information, i.e., video stream. Thus, it is not straightforwardly applicable to our task.

The proposed model is an extension of DeepConvServ. Different from the previous works, the hazard function of our model is defined as the output of the CRNN. This enables us to treat video stream data in the survival analysis framework. Furthermore, our model can predict the lead time considering not only the time-series changes in video stream but also location information.

\section{PROBLEM DEFINITION}

We describe the data considered in this paper and define our task as predicting the lead time of accidents.

Event Recorder Data. First, we define event recorder data as follows. Let $i=1, \ldots, I$ be an index of video data and $V_{i}=\left\{\left(\boldsymbol{x}_{i j}, t_{i j}, l_{i j}\right) \mid\right.$ $\left.j=1, \ldots, J_{i}\right\}$ be the $i$-th video data, where this data is a sequence of triples that contains image $\boldsymbol{x}_{i j}$, time stamp $t_{i j}$ and location $l_{i j}$. Here, $j=1, \ldots, J_{i}$ is a video frame index. Image $x_{i j}$ is taken by the dashcam at time $t_{i j} . l_{i j}$ is the location point represented by its coordinates (i.e., latitude and longitude).

Event Time Data. We then define event time data, which is the time at which the event occurred in each event recorder data sequence. Let $\left(T_{i}, \delta_{i}\right)$ be event time data for video $V_{i}$, where $T_{i}=$ $t_{i J_{i}}-t_{i 1}$ is the lead time from the start of observation to the occurrence of an event or end of observation and $\delta_{i} \in\{1,0\}$ is an indicator variable that is $\delta_{i}=1$ if the video $V_{i}$ contains an event, $\delta_{i}=0$ otherwise. This definition assumes that there is at most one event in one event recorder data sequence. It can be easily applied to the case where several events occur by dividing the total video sequence into segments such that each segment has one event at the end of the video frame or none.

Predicting Event Occurrence. Finally, a problem setting in this paper is defined as follows. Our goal is to predict the lead time of accidents. Let $V^{*}=\left\{\left(\boldsymbol{x}_{j}^{*}, t_{j}^{*}, l_{j}^{*}\right) \mid j=1, \ldots, J^{*}\right\}$ denote the video from the start of driving to the present. Given $V^{*}$, we would like to estimate the lead time $\Delta_{J^{*}}^{*}=t-t_{j}^{*}$ to the occurrence of an event in the future, where $t$ is the time at which an accident will occur.

\section{PRELIMINARY}

Our proposed model is based on survival analysis, which is widely used for estimating event occurrence times. In the following, we briefly describe the survival analysis framework [3].

Survival analysis assumes that the probability of event occurrences is determined by the hazard function which is designed to capture underlying dynamics. The hazard function is defined by

$$
h(\Delta \mid V)=\lim _{d \Delta \rightarrow 0}\left[\frac{\mathrm{P}(\Delta \leq t<\Delta+d \Delta \mid t \geq \Delta)}{d \Delta}\right],
$$

where $d \Delta$ is a small interval around time $\Delta$ and, $\mathrm{P}(\bullet)$ in Eq. (1) is the probability that an event will occur between $\Delta$ and $\Delta+d \Delta$. The hazard function $h(\Delta \mid V) \geq 0$ represents the probability that an event will occur at $\Delta+d \Delta$ immediately after the event has not occurred up to time $\Delta$. Given the observations $\left\{\Delta_{i}\right\}_{i=1}^{I}$, the likelihood is given by

$\mathrm{P}\left(\left\{\Delta_{i}\right\}_{i=1}^{I} \mid h(\Delta \mid V)\right)=\prod_{i=1}^{I}\left\{\left[h\left(\Delta_{i} \mid V_{i}\right)\right]^{\delta_{i}} \cdot \exp \left[-\int_{0}^{\Delta_{i}} h\left(u \mid V_{i}\right) d u\right]\right\}$.

The model parameters can be estimated by maximizing Eq. (2).

The survival function is the probability that an event will not occur before time $\Delta$; it is calculated by $S(\Delta \mid V)=\exp \left[-\int_{0}^{\Delta} h(u \mid V) d u\right]$. 


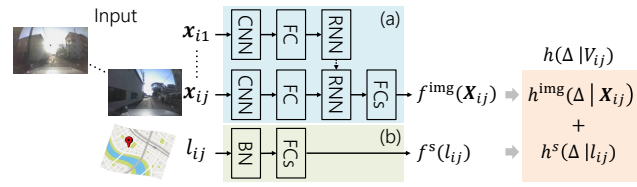

Figure 2: Network structures for (a) video and (b) location.

Predicted lead time $\Delta$ can be determined by thinning [8], a predefined threshold and so on. In this paper, we make predictions based on the expected value of event occurrence time, that is, $\mathbb{E}[h(\Delta \mid V) \cdot S(\Delta \mid V)]$. Here, $h(\Delta \mid V) \cdot S(\Delta \mid V)$ represents the probability that an event will occur at $\Delta+d \Delta$ immediately after the event has not occurred until time $\Delta$.

\section{PROPOSED METHOD}

\subsection{Model Formulation}

We propose a model based on survival analysis to consider two factors (i.e., video and location). We expect the video to have signs of the event e.g., movement of pedestrians around. Furthermore, we expect that the car's current location also has important information. For example, there is a high possibility that children run into a street when there is a school around the current location. Thus, the hazard function is modeled by a combination of two terms,

$$
h(\Delta \mid V)=h^{\mathrm{img}}(\Delta \mid X)+h^{\mathrm{s}}(\Delta \mid l),
$$

where $V$ is a video, $X$ composed of the first to last images included in $V, l$ is the location at the end time of the video. Here, $h^{\mathrm{img}}(\Delta \mid X)$ represents the hazard function that is determined by time-series changes of own car and its surroundings and $h^{\mathrm{s}}(\Delta \mid l)$ represents the hazard function that is determined by the location characteristics. The details of them are described in the following paragraphs.

Hazard Function for Video. We describe the hazard function $h^{\mathrm{img}}(\Delta \mid X)$ for video. This function receives a video stream (i.e., timeseries images) as input. We design the function by using the DNN whose network structure is shown in Figure 2a. Then, $h^{\mathrm{img}}(\Delta \mid X)$ is given by

$$
h^{\mathrm{img}}(\Delta \mid X)=\exp \left[f^{\mathrm{img}}\left(\boldsymbol{X} ; \theta^{\mathrm{img}}\right)\right]
$$

where $f^{\mathrm{img}}\left(\boldsymbol{X} ; \theta^{\mathrm{img}}\right)$ is the function that outputs a scalar value calculated by DNN when receiving an input video. The network comprises a CNN layer for extracting image features, a full connect (FC) layer for compressing the dimensions of image features, an RNN layer for capturing time-series information, and FC layers for converting the output of the RNN layer into scalar values $\left(f^{\mathrm{img}}\left(X ; \theta^{\mathrm{img}}\right)\right)$. The $\mathrm{CNN}$ layer and $\mathrm{RNN}$ layer can use any network structure such as VGG [10] or gated recurrent unit (GRU) [2]. For simplicity, it is assumed that hazard function takes a constant value regardless of lead time $\Delta$.

Hazard Function for Location. We describe the hazard function $h^{\mathrm{s}}(\Delta \mid l)$ for location data. In this paper, we simply use the neural network shown in Figure $2 \mathrm{~b}$ and define it as,

$$
h^{\mathrm{s}}(\Delta \mid l)=\exp \left[f^{\mathrm{s}}\left(l ; \theta^{\mathrm{s}}\right)\right] .
$$

For location, latitude and longitude are normalized in the batch normalization (BN) layer [5]. The FC layers convert the output of the embedding layer into scalar values $\left(f^{\mathrm{s}}\left(l ; \theta^{\mathrm{s}}\right)\right)$.

\subsection{Parameter Estimation}

Each parameter is estimated by backpropagation using the negative log-likelihood as the loss function. From Eq. (2), the likelihood of our model can be written by

$\mathrm{P}(\mathbb{D} \mid h(\Delta \mid V))=\prod_{i=1}^{I} \prod_{j=1}^{J_{i}}\left\{\left[h\left(\Delta_{i j} \mid V_{i j}\right)\right]^{\delta_{i}} \cdot \exp \left[-\int_{0}^{\Delta_{i j}} h\left(u \mid V_{i j}\right) d u\right]\right\}$,

where $\mathbb{D}=\left\{T_{i} \mid i=1, \ldots, I\right\}$ is an event time set, $\Delta_{i j}=T_{i}-t_{i j}$ is the lead time from $t_{i j}$ to the occurrence of an event or censoring and $V_{i j}=\left\{\left(x_{i 1}, t_{i 1}, l_{i 1}\right), \ldots,\left(x_{i j}, t_{i j}, l_{i j}\right)\right\}$ is the video until $t_{i j}$. It is expected to yield accurate predictions from any time point because we use the product of the likelihoods at every time point. Here, the integral part can be calculated by $\int_{0}^{\Delta_{i j}} h\left(u \mid V_{i j}\right) d u=h\left(u \mid V_{i j}\right) \Delta_{i j}$, since $f^{\text {img }}$ and $f^{\mathrm{s}}$ do not depend on $u$.

\section{EXPERIMENTS}

\subsection{Datasets}

We use a real-world dataset to analyze the effectiveness of the proposed model. Our final goal is to predict the lead time of the next accident, however, it is difficult to accumulate large-scale data sets with sufficient numbers of accidents. We instead used a nearmiss incident database [9], which is actually used for verifying the traffic accident anticipation system proposed in [11]. Nearmiss incidents are not accidents, but they do indicate dangerous situations for which responses are needed. We randomly extracted data of 1,800 near-miss incidents and 1,800 safe data (i.e., does not contain near-miss incidents) from the database [9]. Each data was collected by taxis in Shizuoka prefecture in Japan if the longitudinal acceleration/deceleration exceeded $0.45 \mathrm{G}$ (i.e., this is the trigger). Each data contained a 15 second long 30 fps video; 10 seconds before the trigger and 5 seconds after the trigger. We take the trigger time to be the event (near-miss incident) occurrence time. In data preprocessing, we extracted subsequences of random length from 1 to 10 seconds with the trigger as the tail for each near-miss data. This is because each near-miss data needs to include a trigger and to make it impossible to predict the trigger timing from the data length. Also, we randomly extracted subsequences of random length from 1 to 15 seconds from each safe data. This is because safe data do not contain an event. We randomly divided the dataset into three sets: $60 \%$ data for learning, $20 \%$ data for validation, and the remainder for evaluation.

\subsection{Evaluation Metrics}

We used the following two metrics for evaluating the accuracy of prediction. The first metric is capped mean absolute error (CMAE). CMAE evaluates the difference between the predicted lead time and the actual lead time. Unlike mean absolute error, CMAE sets an upper limit on the predicted values, and is represented by CMAE = $\frac{\sum_{(i, j) \in \mathbb{U}}\left|\Delta_{i j}-\min \left(\hat{\Delta}_{i j}, \tau\right)\right|}{|\mathbb{U}|}$, where $\mathbb{U}$ is a set of every timing of uncensored data, that is, near-miss incident data. Here $\tau$ is a threshold for setting the upper limit of the predicted value. We set it to 15 seconds because no data sequence exceeded 15 seconds, and higher values meant "no event occurred". CMAE cannot evaluate the accuracy of predicting for safe data i.e., whether the model catches the state 
Table 1: C-index and CMAE for near-miss prediction.

\begin{tabular}{lcc}
\hline & c-index & CMAE (seconds) \\
\hline ClassfierBase & 0.649 & 3.82 \\
DeepConvSurv & 0.661 & 3.73 \\
Proposed1 (w/o location data) & 0.686 & 3.42 \\
Proposed2 (w/ location data) & 0.687 & 3.29 \\
\hline
\end{tabular}

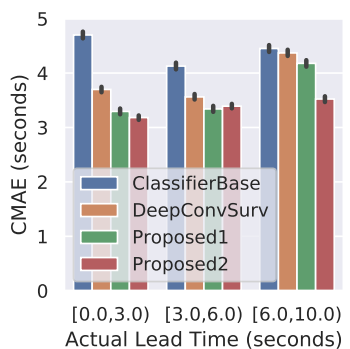

(a)

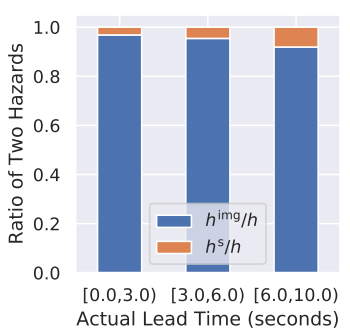

(b)
Figure 3: (a) CMAE versus actual lead time. (b) Ratio of $h^{\text {img }}$ and $h^{\mathrm{s}}$ versus actual lead time.

that the event does not occur for some time. Therefore, we use the time-dependent concordance index (c-index) as the other metric [4]. The c-index is commonly used in the evaluation of survival analysis techniques, which indicates how well the model can order the time of occurrence of events for all data. Smaller CMAE values and larger c-index values (up to 1) indicate better prediction accuracy.

\subsection{Compared Models}

We compare our models with two deep learning models.

ClassifierBase is a multi-class classification model we prepared for evaluation. It receives only images as input and outputs a class label that indicates the lead time to the event occurrence. As target classes, we prepared 11 classes consisting of 10 near-miss classes and 1 safe class. Each of near-miss class is obtained by dividing 10 seconds, the period before the trigger, into 1-second units. For calculating CMAE, we used the median of each class label, i.e., $0.0-$ 1.0 seconds range class is $0.5,1.0-2.0$ seconds range class is $1.5, \ldots$, and 10.0 seconds or more class (safe class) is $\tau=15.0$ seconds.

DeepConvSurv is a model based on survival analysis [14]. It takes only an image $\boldsymbol{x}_{J^{*}}^{*}$ as input and predicts the lead time to the event occurrence.

Proposed 1 is our proposed model without location data. It takes only images $\left\{\boldsymbol{x}_{j}^{*} \mid j=1, \ldots, J^{*}\right\}$ as input and predicts the lead time to the event occurrence.

Proposed 2 is our proposed model as described in Section 5. It takes images and location data $\left\{\left(\boldsymbol{x}_{j}^{*}, l_{j}^{*}\right) \mid j=1, \ldots, J^{*}\right\}$.

We used the VGG that was trained on Places365 data [13] for the CNN part and used adabound [7] as the optimizer. We determined hyperparameters such as the number of units and the learning rate of the optimizer by using the validation data based on c-index.

\subsection{Results}

Table 1 lists the results attained. It shows that the Proposed 1 is superior to the baseline models in terms of both metrics. Also, Proposed2 is superior to Proposed1. These confirm that both RNN and location data do improve the accuracy.
Figure 3a plots CMAE versus actual lead time (i.e., $\Delta_{i j}$ ). If actual lead time is from 0 to 3 seconds, sudden braking is necessary, if 3 seconds to 6 seconds, deceleration is advised, and if it is 6 seconds or more, a warning to the driver is advised, which forms the appropriate ADAS responses. This result shows that CMAE improves with closeness to the accident closer to the event. However, in Proposed2, the deterioration of CMAE with greater actual lead times is moderate. This indicates that the cause of the near-miss incident is not shown in the video at long lead times, and it is difficult to predict from the video. On the other hand, the location data is effective in enhancing prediction performance in such cases. Figure $3 \mathrm{~b}$ plots the ratio of two hazards for three actual lead time ranges. The ratio was calculated by dividing the average of $h^{\mathrm{img}}$ or $h^{\mathrm{s}}$ by the average of $h$. The above is confirmed because as this result Figure $3 \mathrm{~b}$ shows that the contribution of $h^{\mathrm{s}}$ is larger at 6-10 seconds than at $0-3$ seconds.

\section{CONCLUSIONS}

In this paper, we tackle the task of predicting the lead time of an accident between own car and another traffic obstacle based on dashcam video and location data. We focused on survival analysis and extended it to handle video streams (i.e., high dimensional time series features) by incorporating RNN. Furthermore, our proposal incorporates location features. Experiments on real-world data showed that the two factors are effective in improving the accuracy of lead time determination. Our future work is to verify with real-world accident data and to add information sources such as sensors and map-based data; it is useful for yielding better prediction results.

\section{REFERENCES}

[1] Fu-Hsiang Chan, Yu-Ting Chen, Yu Xiang, and Min Sun. 2016. Anticipating Accidents in Dashcam Videos. In ACCV. 136-153.

[2] Kyunghyun Cho, Bart Van Merriënboer, Caglar Gulcehre, Dzmitry Bahdanau, Fethi Bougares, Holger Schwenk, and Yoshua Bengio. 2014. Learning Phrase Representations using RNN Encoder-Decoder for Statistical Machine Translation. arXiv: 1406.1078

[3] David Collett. 2003. Modelling Survival Data in Medical Research. Chapman and Hall/CRC.

[4] Frank E Harrell, Kerry L Lee, and Daniel B Mark. 1996. Multivariable Prognostic Models: Issues in Developing Models, Evaluating Assumptions and Adequacy, and Measuring and Reducing Errors. Statistics in Medicine 15, 4 (1996), 361-387.

[5] Sergey Ioffe and Christian Szegedy. 2015. Batch Normalization: Accelerating Deep Network Training by Reducing Internal Covariate Shift. arXiv:1502.03167

[6] How Jing and Alexander J Smola. 2017. Neural Survival Recommender. In WSDM. ACM, 515-524.

[7] Liangchen Luo, Yuanhao Xiong, Yan Liu, and Xu Sun. 2019. Adaptive Gradient Methods with Dynamic Bound of Learning Rate. In ICLR. New Orleans, Louisiana.

[8] Yosihiko Ogata. 1981. On Lewis' Simulation Method for Point Processes. IEEE Trans. Inf. Theory 27, 1, 23-31.

[9] Pongsathorn Raksincharoensak. 2013. Drive Recorder Database for Accident/Incident Study and Its Potential for Active Safety Development. http: //web.tuat.ac.jp/ smrc/pdf/Driverecorder.pdf.

[10] Karen Simonyan and Andrew Zisserman. 2014. Very Deep Convolutional Networks for Large-Scale Image Recognition. CoRR abs/1409.1556 (2014).

[11] Tomoyuki Suzuki, Hirokatsu Kataoka, Yoshimitsu Aoki, and Yutaka Satoh. 2018. Anticipating Traffic Accidents with Adaptive Loss and Large-scale Incident DB. arXiv: 1804.02675

[12] Jiawen Yao, Xinliang Zhu, Feiyun Zhu, and Junzhou Huang. 2017. Deep Correlational Learning for Survival Prediction from Multi-modality Data. In MICCAI. Springer, 406-414.

[13] Bolei Zhou, Agata Lapedriza, Aditya Khosla, Aude Oliva, and Antonio Torralba. 2017. Places: A 10 million Image Database for Scene Recognition. In TPAMI.

[14] Xinliang Zhu, Jiawen Yao, and Junzhou Huang. 2016. Deep Convolutional Neural Network for Survival Analysis with Pathological Images. In BIBM. IEEE, 544-547.

[15] Xinliang Zhu, Jiawen Yao, Feiyun Zhu, and Junzhou Huang. 2017. WSISA: Making Survival Prediction From Whole Slide Histopathological Images. In CVPR. 